\title{
The effect of heart disease on pulmonary function
}

\author{
C. W. Hertz \\ M.D. \\ Krankenhaus Mühlenberg, Malente
}

\begin{abstract}
Summary
In pulmonary congestion dyspnoea is due not only to an increase in elastic but also in viscous work. Increased airway resistance can be reduced in most of these cases by inhalation of adrenergic or anticholinergic agents. Therefore, besides bronchial mucosal swelling induced by congestion in the bronchial vessels via bronchopulmonary anastomoses, bronchospasm can take place and lead to cardiac asthma. The possible genesis of this bronchoconstriction is discussed.
\end{abstract}

THE close relationship between cardiac and respiratory function is apparent since cardiac and pulmonary disorders can lead to the same symptom, namely dyspnoea. Heart diseases with impaired blood drainage from the lung show effects similar to the bronchopneumopathies but the extent to which pulmonary congestion affects respiratory mechanics has not been determined.

The cardiovascular system and the lung are one functional unit. The heart is an auxiliary organ for respiration in that it is the machine which renders the respiratory function of the lung effective in the peripheral areas of the body. The pulmonary circulation is perfused by the same blood flow volume as the systemic circulation; however, it differs in essential aspects from the latter.

The mean pressure in the pulmonary artery is normally approximately one-sixth of the mean arterial pressure in the systemic circulation, and the resistance in the pulmonary circulation is approximately one-tenth of that in the systemic circulation. There are no valves in the pulmonary veins; this fact is of particular importance in connection with pulmonary hypertension in left venticular failure or mitral stenosis. If the blood from the lung is prevented from draining, the pulmonary arterial pressure is increased as a consequence of the increased pulmonary venous pressure. By contrast, in the systemic circulation a venous drainage block of the right atrium does not have any noteworthy effect on

Correspondence: Professor C. W. Hertz, Department of Internal Medicine, Krankenhaus Mühlenberg, D 2427 Malente, Federal Republic of Germany. arterial blood pressure. Whereas the majority of arterial hypertensions are so-called essential hypertensions, pulmonary hypertension is almost exclusively secondary to a heart or lung disorder. Only the consequences of blood drainage impairments secondary to heart disease will be considered in this paper.

Because of its low-pressure system the pulmonary circulation is more dependent upon external influences than the systemic circulation. Two factors are important for the distribution of blood in the lung.

As early as 1934, Björkman showed by means of bronchospirometry that when a person lies on his side there is a considerable increase in pulmonary blood flow in the lower side (Björkman, 1934). In 1953, Martin, Cline and Marshall found by means of gas analyses from various bronchi a decrease in the ventilation-perfusion ratio from top to bottom when the body was in an upright position and hence a relatively increased blood flow in the base of the lung (Martin, Cline and Marshall, 1953). This was later confirmed by West and Dollery (1960) using a radioactive tracer. When pulmonary blood flow is increased, i.e. during physical exercise, but also in pulmonary congestion due to increased pulmonary venous pressure there is increased perfusion of the lung apex. Dollery and West (1960) showed that in pulmonary congestion the situation can occur in which the upper parts of the lung are better perfused than the lower parts, possibly as a result of interstitial oedema in the lower sections of the lung.

In addition to hydrostatic pressure, the relationship between intrapulmonary vascular pressure and alveolar pressure also plays a part in pulmonary blood distribution. If, for example, the alveolar pressure exceeds the pulmonary venous pressure, the pulmonary blood flow is determined more by the pulmonary arterial/alveolar pressure difference than by the arteriovenous pressure difference. Thus, whereas obstructive bronchopneumopathies are often associated with increased alveolar pressure, heart diseases involving pulmonary congestion result $\mathrm{n}$ the reverse case, i.e. increased pulmonary venous pressure and a more homogeneous distribution of blood within the lung. 


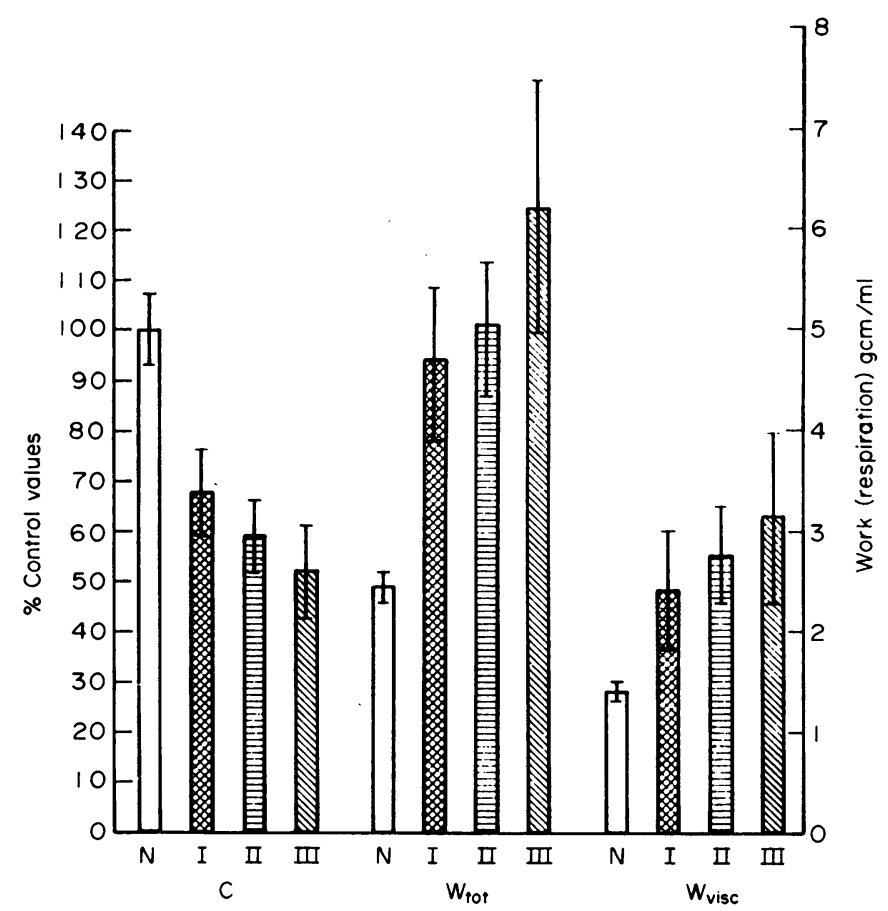

FIG. 1. Mean values \pm 2 s.e. mean of the compliance $(C)$, total inspiratory work $\left(W_{\text {tot }}\right)$, and viscous work ( $\left.W_{\text {visc }}\right)$ in healthy subjects $(N)$, and with three grades of mitral stenosis (N.Y. Heart Association Classification). Number of patients in each group: N, 12; I, 30; II, 43; III, 27. All values expressed as percentage of healthy controls. Work expressed as $\mathrm{gcm} / \mathrm{ml}$.

However, it is known that the distribution of pulmonary blood flow is not only dependent upon these mechanical factors but also on local oxygen pressures. Owing to a marked regional lowering of the alveolar oxygen pressure there is a local increase in vascular resistance and, hence, diversion of blood into better perfused parts. This mechanism may also play a role in pulmonary congestion because of a local shift of the ventilation : perfusion ratio which may give rise to vasoconstriction. The effect of local hypoxia on blood flow distribution was shown 20 years ago by means of bronchospirometry with unilateral re-breathing to result in a decrease in blood flow of approximately $30 \%$ (Hertz, 1955, 1956a).

There are few studies on the effect of local $\mathrm{PCO}_{2}$ increase in man. The effects of a local $\mathrm{PCO}_{2}$ increase, however, will always be less than those of local hypoxia since the local $\mathrm{CO}_{2}$ pressure cannot exceed that of the mixed venous blood, and this is only slightly greater than that of the arterial blood.

Unilateral re-breathing will result in a difference in alveolar $\mathrm{CO}_{2}$ pressure between the two sides of the lung of 5-15 mm Hg compared with a difference of
$>50 \mathrm{~mm}$ in alveolar oxygen pressure in unilateral hypoxia. The effect of unilateral $\mathrm{CO}_{2}$ increase on blood flow distribution due to re-breathing has been studied using bronchospirometry with oxygen respiration (Hertz, 1956b). In thirty-two studies in twenty patients the mean blood flow in the $\mathrm{CO}_{2}$ rebreathing side decreased to $93.6 \%$ of the baseline value. No greater effects can be anticipated under physiological conditions because no marked local $\mathrm{PCO}_{2}$ increases can be expected in distribution disorders. General hypercapnia is a different matter; but this is not expected in functional disorders of cardiac aetiology.

Although mechanical forces and gas pressures have an effect on pulmonary blood flow, the reverse can occur, in which case changes in blood flow have an effect on lung function.

In left heart failure, resulting either from an increase in pressure in the left atrium or an increase in end-diastolic pressure in the left ventricle, there is vascular congestion of the lung which has an effect on respiratory mechanics. The elastic properties of the lung are dependent upon all components of the lung tissue, including the blood in the pulmonary 


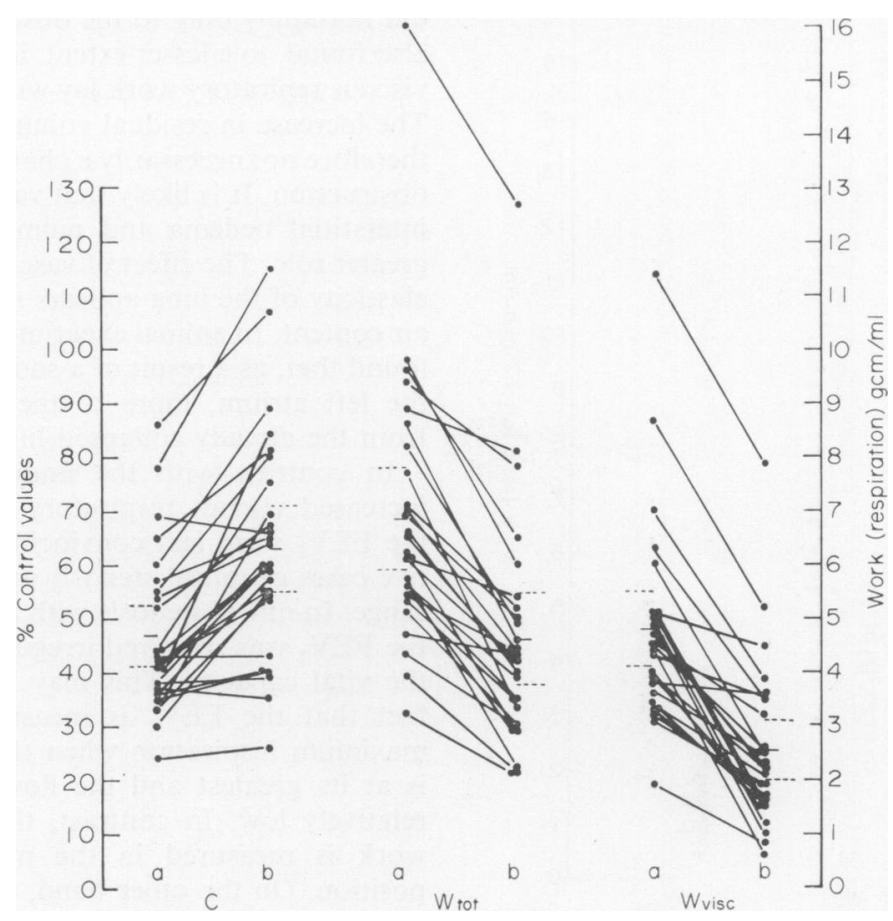

Fig. 2. Individual values together with mean \pm s.e. mean for compliance (C), total inspiratory work ( $W_{\text {tot }}$ ), and viscous work of breathing (Wvisc) before (a) and after (b) inhalation of orciprenaline in twenty-seven cases of mitral stenosis with bronchial obstruction (after Hamm and Schölmerich, 1964).

vessels, so that blood congestion must result in a reduction in static compliance. As early as 1888 , von Basch described experiments which showed a reduced compliance of the lungs in vascular congestion (von Basch, 1888).

Several authors have reported a restrictive ventilatory disorder in left heart failure and reduced vital capacity. Thus, in the twenties, daily measurement of vital capacity in left heart failure was used as an indicator of therapeutic success.

The conditions in pulmonary congestion suggest that increase in respiratory work is required to overcome elastic resistance. Mitral stenosis is well suited for the study of this problem since the haemodynamic disturbance is relatively constant. Studies of the mechanics of respiration have shown that respiratory work increases not only to overcome elastic resistance but also to overcome viscous resistances. Figure 1 shows the results of studies in 100 operated, and therefore confirmed, cases of mitral stenosis by Hamm and Schölmerich (1964). On the left are the mean values for compliance as a percentage of the expected value as found in twelve healthy volunteers, thirty patients with mitral stenosis stage I (New York Association classification) forty-three patients in stage II and forty-seven patients in stage III. A reduced compliance was found which was related to the severity of the disease. The main cause for the increased elastance is, above all, the blood volume in the lung, but other factors such as interstitial oedema, fibrosis, possibly small areas of atelectasis, swelling of the bronchial mucosa, and changes in alveolar surface tension, may also play a part.

As a result of the reduction in compliance, the work of breathing was increased, depending upon the degree of severity of the clinical symptoms of mitral stenosis. In approximately $40 \%$ of the patients with mitral stenosis the viscous work had also increased above normal values, again dependent upon the degree of severity of the disorder (Fig. 1, column 3).

The increased viscous work is probably primarily the result of bronchial obstruction. Thus the total and viscous respiratory work decreases and the dynamic compliance increases after the inhalation of orciprenaline (Fig. 2). In most cases, the viscous respiratory work does not return to normal. The 


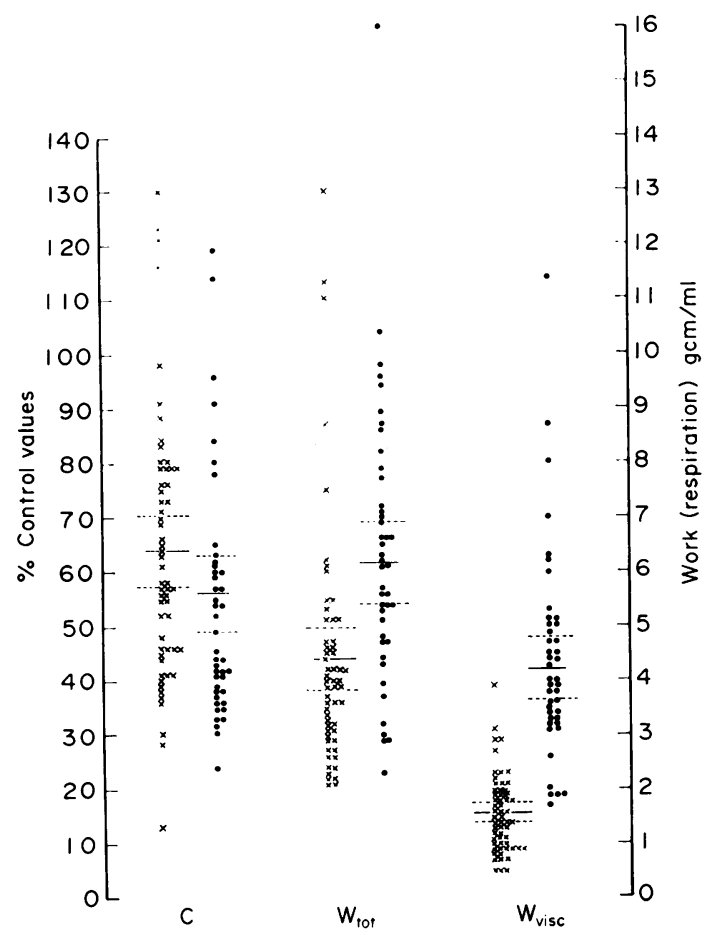

Fig. 3. Individual values together with mean \pm s.e. mean for compliance (C), total inspiratory work ( $W_{\text {tot }}$ ), and viscous work of breathing $\left(W_{\text {visc }}\right)$ in fifty-seven cases of mitral stenosis with pulmonary restriction and forty-three cases with additional bronchial obstruction (after Hamm and Schölmerich, 1964).

increase in dynamic compliance is probably also attributable to the reduction of obstruction since the dynamic compliance in obstruction does not correspond to the static compliance. The obstruction which can be deduced from the results of this study is of primary importance. As a result of the increased vascular congestion of the lung the tissue frictional resistance may also contribute to the increase in viscous respiratory work. Hysteresis is only likely to be of importance in extreme pulmonary congestion and in the oedematous lung as a result of altered surface tensions.

Figure 3, also taken from the study by Hamm and Schölmerich (1964), presents data for cases divided into restrictive and obstructive groups. Cases termed obstructive were those which showed a clear response to orciprenaline as well as those in which the ratio of viscous : elastic work was $>0.75$. The difference in the mean values for percentage compliance were not significant, whereas the differences in the total and, above all, the viscous work of breathing were highly significant.

In most cases of mitral stenosis there was an increase in residual volume. However, this increase did not apply only to the obstructive cases, but was also found, to a lesser extent, in those patients whose viscous repiratory work lay within the normal range. The increase in residual volume in mitral stenosis is therefore not necessarily a characteristic of bronchial obstruction. It is likely that vascular congestion and interstitial oedema and pulmonary fibrosis play a greater role. The effect of vascular congestion on the elasticity of the lung appears to be a function of the air content. In animal experiments Borst et al. (1957) found that, as a result of a sudden fall in pressure in the left atrium, more of the residual air escaped from the already collapsed lungs.

In contrast with the findings in patients with increased viscous respiratory work, the changes in the $\mathrm{FEV}_{1}$ were less convincing. The purely restrictive cases of mitral stenosis were within the normal range. In mitral stenosis with additional obstruction on the FEV 1 was scattered irregularly down to $30 \%$ of the vital capacity. This may have been due to the fact that the $\mathrm{FEV}_{1}$ is measured at the point of maximum inspiration when the bronchial diameter is at its greatest and the flow resistance therefore relatively low. In contrast, the viscous respiratory work is measured in the normal end-expiratory position. On the other hand, our own studies, with values for viscous respiratory work of approximatels $4 \mathrm{gcm} / \mathrm{ml}$, there was no demonstrable relationshi with relative $\mathrm{FEV}_{1}$ (Ghosh and Hertz, 1971). As has already been shown, the mean values for viscous? respiratory work lay between 2.5 and $3.2 \mathrm{gcm} / \mathrm{ml}$ in all cases of mitral stenosis investigated by Hamm and Schölmerich (1964). Only in twenty patients was the viscous respiratory work greater than $4 \mathrm{gcm} / \mathrm{ml}$ and accordingly the $\mathrm{FEV}_{1}$ in twenty-three patients was less than $65 \%$.

The finding that left heart failure can cause an airway obstruction was confirmed using body plethysmography. Figure 4 shows the results of Kammler et al. (1968) in fifteen healthy control subjects (I), twenty-seven patients with heart disease, who complained of dyspnoea on fairly vigorous exercise (II), and thirty-five patients with heart disease with dyspnoea on light exercise (III). In many cases, especially in those with more severe heart disease, there was a slight to moderate increase in airway resistance. Here again, as Wettengel (1974) showed in seven patients with mitral stenosis, a or reduction in resistance was achieved by inhalation $N$ of an adrenergic and anticholinergic agent. Within $\underset{\omega}{\mathrm{W}}$ $30 \mathrm{~min}$ of inhalation of an atropine derivative, resistance fell by $36 \%$ and, after orciprenaline, by $28 \%$.

It has thus been shown that in many patients with pulmonary congestion, there is bronchial obstruc- $T$ tion. These results are in agreement with the clinical findings, for example in congestive bronchitis. 


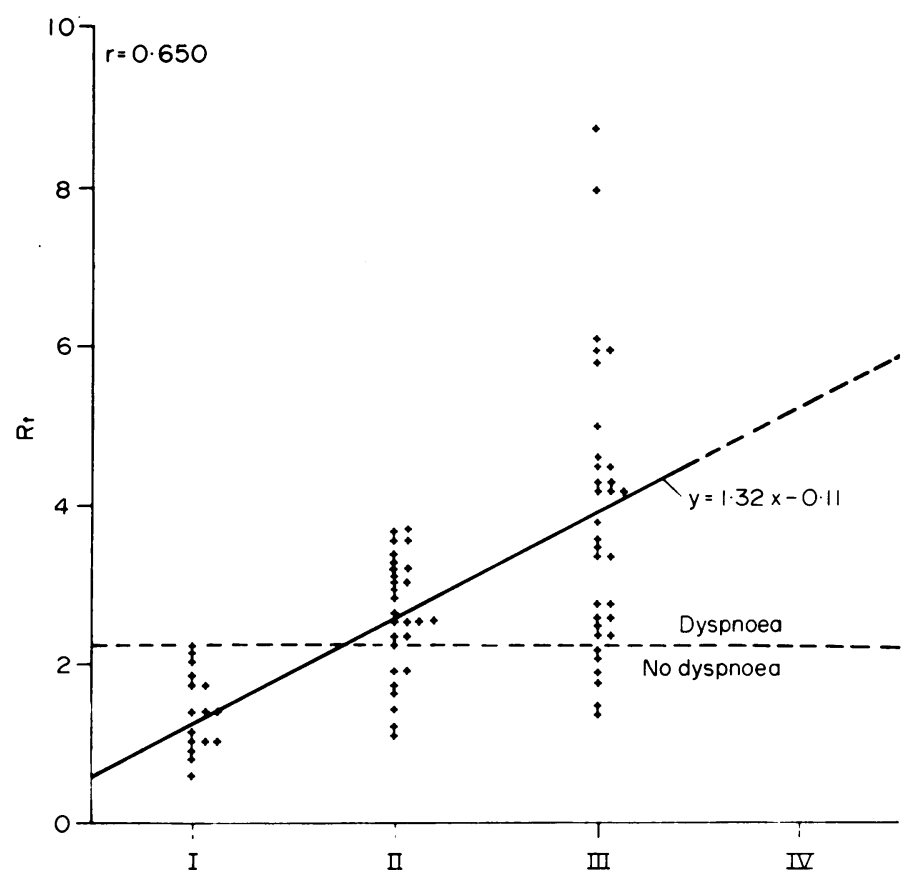

Fig. 4. Airway resistance $\left(R_{\mathbf{t}}\right)$ in cardiac patients. $I=$ healthy control subjects $(n=15)$, II $=$ patients with heart disease, who complained of dyspnoea on fairly vigorous exercise $(n=27)$, III = cardiac patients with dyspnoea on light exercise $(\mathrm{n}=35)$ (after Kammler et al., 1968).

Because the blood from the bronchial arteries drains into the pulmonary veins via the broncho-pulmonary capillary network, there is congestion in the pulmonary veins as well as in the bronchial circulation. This can lead to an oedema of the bronchial wall with concomitant constriction of the lumen and transudation into the bronchial lumen. The bronchial and pulmonary arteries, like the bronchial and pulmonary veins, are connected with each other by anastomoses so that the two circulations are closely connected. Morphologically, several authors have found an enlargement of all parts of the bronchial vascular system in mitral stenosis.

Apart from this constriction of the bronchial lumen due to mucosal swelling, bronchospasm may also play a part in the obstruction caused by left heart failure. Thus, sudden attacks of respiratory distress and wheezing induced by acute pulmonary congestion are referred to as cardiac asthma. Pulmonary oedema can also be heralded by clinical and auscultatory findings which resemble those of status asthmaticus. This can give rise to greater resistance values than those described. However, in the cases of mitral stenosis, the obstruction was reversible on inhalation of a bronchospasmolytic agent, which seems to show that there is a broncho-constrictor component.
So far, there have only been hypotheses about the possible genesis of this bronchoconstriction in pulmonary congestion. Perhaps the congestion gives rise to metabolites in the bronchial mucosa which cause bronchoconstriction. It was demonstrated in animal experiments that irritant receptors can be stimulated by pulmonary congestion. An increase in airway resistance was demonstrated when the pressure in the left atrium was rapidly increased and this could be abolished by vagotomy or atropine. The extreme retardation in flow or blood stasis in certain areas of the lung in advanced pulmonary congestion may be of importance. Following blockade of one branch of the pulmonary artery, there was a reduction in respiratory volume in the same side of the lung, which could only be explained by bronchoconstriction. The blood in the dependent vessels stagnates and there is a diminution in tidal volume and, incidentally, also in respiratory rate (Hertz et al., 1962). The addition of $\mathrm{CO}_{2}$ to the respired air of the blocked side not only abolished the reduction in rate but also the unilateral reduction in tidal volume. One can speculate whether local hypocapnia or even acapnia is to some extent responsible for this and the effect of the addition of $\mathrm{CO}_{2}$ tends to support this. For, wherever blood was stagnating in the pulmonary capillaries with maintained venti- 
lation the $\mathrm{CO}_{2}$ pressure was reduced within a very short time to values approaching zero (Hertz et al., 1962).

Finally, in advanced pulmonary congestion, especially in mitral stenosis, several investigators have demonstrated a reduction in diffusion capacity. This showed that there is a clear correlation with the degree of severity of the underlying heart disease.

The main causes of dyspnoea in heart disease are to be found primarily in the increased elastic work of breathing as a result of the reduced compliance with overfilling of the pulmonary blood vessels and, secondly, in the obstruction, which plays a prominent role in cardiac asthma. The reduction in diffusion capacity in the development of dyspnoea plays, at most, only a subordinate role.

Congestive bronchitis with early pulmonary oedema, and the severe respiratory insufficiency in chronic bronchitis can show the same clinical picture. However, with a view to therapy, it is of the utmost importance to differentiate between them. If a patient presents with advanced dyspnoea and rhonchi over the lung areas and pulmonary oedema is erroneously diagnosed, the administration of oxygen and morphine could have deleterious effects. This is because morphine acts as a respiratory depressant and the administration of oxygen can lead to $\mathrm{CO}_{2}$ narcosis, especially if advanced respiratory acidosis already exists. For purposes of differential diagnosis, blood gas analysis and X-ray can be of assistance. With the modern specialization of medicine it is important for the chest physician to be aware of left heart failure and for the cardiologist to be aware of respiratory insufficiency.

\section{References}

BASCH, S. vON (1888) Über Lungenschwellung und Lungenstarrheit. Wiener medizinische Presse, 14, 587.
BjörkmaN, S. (1934) Bronchospirometrie. Eine Methode, die气̂ Funktion der menschlichen Lunge getrennt und gleich- $\bar{z}$ zeitig zu untersuchen. Acta medica scandinavica, Suppl. 56, 1.

Borst, H.G., Berglund, E., Whittenberger, J.L., Mead, J., McGregor, M. \& Collier, C. (1957) The effects of pulmonary vascular pressures on the mechanical properties of the lungs of anesthetized dogs. Journal of Clinical $\bar{C}$ Investigation, 36, 1708.

DOLLERY, C.T. \& WEST, J.B. (1960) Regional uptake of radioactive oxygen and carbon dioxide in the lungs of $\vec{\Phi}$ patients with mitral stenosis. Circulation Research, 8, 765.

Ghosh, S.K. \& HerTZ, C.W. (1971) Die Beziehungen zwischen Sekundenkapazität und anderen atemmechani-schen Größen. Klinische Wochenschrift. 49, 1083.

HaMm, J. \& SCHÖLmerich, P. (1964) 'Über Lungenschwellung und Lungenstarrheit'. Atemmechanische Untersuchungen $\vec{\omega}$ an 100 Mitralstenosen. Klinische Wochenschrift, 42, 1108.

HerTZ, C.W. (1955) Die Durchblutungsgröße hypoventi lierter Lungenbezirke. Verhandlungen der Deutschen 3 Gesellschaft für Kreislaufforschung, 21, 447.

HeRTZ, C.W. (1956a) Untersuchungen über den Einfluß derN alveolaren Gasdrucke auf die intrapulmonale Durchblu- 9 tungsverteilung. Klinische Wochenschrift, 34, 472.

HeRTZ, C.W. (1956b) Einseitige CO2-Erhöhung und Durch-î blutungsgroße jeder Lungenseite. Klinische Wochenschrift, 34, 532.

Hertz, C.W., Witzleb, E., Freund, H. \& Schlepper, M. 을 (1962) Veränderungen der Atmung bei einseitiger Blockade der A. pulmonalis. Pflügers Archiv für die gesamte $D$ Physiologie des Menschen und der Tiere, 276, 280.

Kammler, E., Reichel, G., Ulmer, W.T., Schürmeyer, E. \& WINDHAGEN, K.E. (1968) Untersuchungen zur pul- $\overrightarrow{0}$ monalen und cardialen Dyspnoe. Verhandlungen \& Deutschen Gesellschaft für innere Medizin, 74, 210.

Martin, C.J., Cline, F. JR \& Marshall, H. (1953) Lobāri alveolar gas concentrations: effect of body position. Journal of Clinical Investigation, 32, 617.

WeST, J.D. \& DollerY, C.T. (1960) Distribution of blood flow and ventilation-perfusion ratio in the lung measured

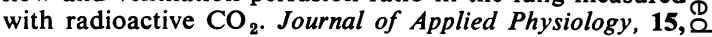
405.

Wettengel, R. (1974) The effects of Sch 1000 MDI in $\bar{\partial}$ patients with mitral stenosis. In: The place of parasympatholytic drugs in the management of chronic obstructive airways disease. International Symposium, Killarney, 21 September 1974. 